\title{
A NONLINEAR STABILITY ANALYSIS OF A MODEL EQUATION FOR ALLOY SOLIDIFICATION
}

BY

\author{
D.J. WOLLKIND \\ D.B. OULTON \\ R. SRIRANGANATHAN
}

IMA Preprint \#17

Apri1 1983

INSTITUTE FOR MATHEMATICS AND ITS APPLICATIONS UNIVERSITY OF MINNESOTA

514 Vincent Hall 206 Church Street SE. Minneapolis, Minnesota 55455 
IMA

Preprint \# Author(s)

1

2 Raphael De laLlave

3

4

5

6

7

8

9

10

11

12

13

14
Henry Simpson
Scott Spector

George R. Sell

Milan Miklavcic

Hans Weinberger

Bau-Sen Du

Hans Weinberger

Lindsay R. Angel

D. Fennell Evans

Barry Ninham

D. Fennell Evans

D.J. Mitchell

Suragit Mukherjee

Barry Ninham

Leif Arkeryd

Luis Magalhaes

Kenneth Meyer

C. Eugene Wayne

\section{Title}

Workshop Summaries from the September 1982 workshop on Statistical Mechanics, Dynamical Systems and Turbulence

A Simple Proof of $C$. Siegel's Center Theorem

On Copositive Matrices and Strong Ellipticity for Isotropic Elastic Materials

Vector Fields in the Vicinity of a Compact Invariant Manifold

Non-linear Stability of Asymptotic Suction

A Simple System with a Continuum of Stable Inhomogeneous Steady States

Period 3 Bifurcation for the Logistic Mapping

Optimal Numerical Approximation of a Linear Operator

Three Component Ionic Microemulsions

Surfactant Diffusion; New Results and Interpretations

A Remark about the Final Aperiodic Regime for Maps on the Interval

Manifolds of Global Solutions of Functional Differential Equations

Tori in Resonance

Surface Models with Nonlocal Potentials: Upper Bounds 


\section{A Nonlinear Stability Analysis of a \\ Model Equation for Alloy Solidification}

D. J. Wollkind

Department of Applied Mathematics, Washington State University, Pullman Washington, 99164-2930, USA

D. B. Oulton

Department of Mathematics, Virginia Wesleyan College, Norfolk, Virginia, 23502, USA

and

R. Sriranganathan

Department of Mathematics, University of Idaho, Moscow, Idaho, 83843, USA 


\section{Abstract}

A weakly nonlinear stability analysis of the Stuart-Watson type is performed on the planar interface solution to the Sivashinsky model equation for dilute binary alloy solidification. The Landau constant appearing in the amplitude equation is calculated by means of both a direct method of solution and the traditional more indirect one of employing the adjoint linear eigenvector. It is concluded that for moving boundary problems of this sort the direct method is superior to the adjoint operator method especially in those instances where the solution itself is desired and not merely just the solvability condition. 


\section{Introduction}

Controlled plane front solidification of alloys and other binary substances under an imposed temperature gradient is used in practice to grow single crystals, refine materials (e.g., zone refining), and obtain uniform or non-uniform composition within the material grown [1]. The most important industrial applications of this type of solidification are for growth of crystals for metal oxide semiconductors (MOS's) [1]. Growth of oxide crystals for jewels is another, much older commercial application of single crystal growth [1]. Another important application is in growth of oxides for laser systems and other optical devices [1]. Further industrial applications arise in ingot casting and in the steel and glass industries [2]. For all of these solidification situations involving binary materials, quantitative predictions of interfacial cellular morphology, including information on cell size and intracellular solute distribution, prove to be extremely valuable and are of a particular aid to industrial researchers.

Recently, in order to explain more fully the morphology and solute distribution of the solid-liquid interface observed during the controlled solidification of a dilute binary alloy, we performed a three-dimensional extension [3] of an existing two-dimensional nonlinear stability analysis [4] appropriate to this problem under those conditions for which a planar interface breaks down and forms cells. Unlike most weakly nonlinear stability analyses [5] in which the Landau-type constants appearing in the amplitude equations [6] are calculated by means of a Fredholm-like solvability condition employing the adjoint linear eigenvector [4-9], we determined those constants by using a more direct approach [3].

Motivated by the philosophy of Segel [10] we wish to introduce a model equation for our system, perform a weakly nonlinear stability analysis of 
its planar interface solution by both the direct and adjoint operator methods, and then compare the results so obtained with each other. For this purpose, we select a model equation for solidification developed by Sivashinsky which is based upon an earlier contribution of Langer's [12]. This equation preserves the essential features of the full system of solidification equations [4] while markedly reducing the complexities involved in our nonlinear analysis. Hence, it is perfect for comparing the two methods under examination in this paper.

In particular after Sivashinsky [11] we consider the following nondimensionalized model equation and boundary conditions for solidification: For $z>\zeta(x, t)$ (in the liquid):

$$
\left[\frac{\partial^{2}}{\partial x^{2}}+\frac{\partial^{2}}{\partial z^{2}}+\frac{\partial}{\partial z}-\frac{\partial}{\partial t}\right] c(x, z, t)=0 .
$$

For $z=\zeta(x, t)$ (at the interface):

$$
\begin{aligned}
& M(C-1)=G \zeta-U \zeta_{x x}\left(1+\zeta_{x}^{2}\right)^{-3 / 2}, \\
& \frac{\partial C}{\partial z}-\zeta_{x} \frac{\partial C}{\partial x}=(k-1) C\left(1+\zeta_{t}\right) .
\end{aligned}
$$

For $z \rightarrow \infty$ (far field condition):

$$
C \rightarrow C_{0}(z)=1+(k-1)\left(1-e^{-z}\right)
$$

Here

$$
(x, z) \equiv \text { a uniformly moving two-dimensional coordinate system such that }
$$
the $x$-axis coincides with the mean position of the solid-liquid interface and the $z$ coordinate is a measure of the distance from that mean position,

$$
\begin{aligned}
& t \equiv \text { time, } \\
& C(x, z, t) \equiv \text { solute concentration, } \\
& \zeta(x, t) \equiv \text { deviation of the interface from its mean planar } \\
& \text { position, }
\end{aligned}
$$




$$
\begin{aligned}
& M \equiv \text { slope of the liquidus line on the phase diagram, } \\
& G \equiv \text { fixed temperature gradient }, \\
& U \equiv \text { uniform rate of solidification, } \\
& k \equiv \text { solute segregation coefficient, }
\end{aligned}
$$

and

$$
C_{0}(z) \equiv \text { planar interface solution of (1.1) and (1.2) corresponding }
$$

to the uniform growth of a planar interface $(\zeta \equiv 0)$ of solid into the molten phase.

Hence, (1.1) represents two-dimensional diffusion of solute in the liquid in the absence of convection. In this model solute diffusion in the solid is neglected and in order to determine the composition of the solid solution adjacent to the interface one uses the relation

$$
C_{S}=k C \text { for } z=\zeta(x, t) \text {. }
$$

Boundary condition (1.2a) can be derived from the Gibbs-Thomson equation for a dilute binary alloy in the absence of interface attachment kinetics, $\delta T$ [4]. In such an instance the latter describes the alteration, $\Delta T$, of the interfacial temperature, $T$, from the equilibrium melting temperature at a planar interface, $T(C)$, due to the curvature, $\kappa$, of the interface itself. That is,

$$
T=T(C)+\Delta T+\delta T \text { for } z=\zeta(x, t) \text {. }
$$

Then defining the above quantities by the simplified conditions

$$
T \equiv T_{0}+G z, T(C) \equiv T_{0}+M(C-1), \Delta T \equiv U_{K} \text {, and } \delta T \equiv 0 \text {, }
$$

yields the desired result. Boundary condition (1.2b) follows from the balance of solute at the interface. A further simplifying assumption in this model is that $z$ extends to infinity. The far field condition of (1.3) is a statement of the fact that far from the interface it is expected that the influence of the shape of that interface on the solute field will become negligible. 
2. The Stuart-Watson method of nonlinear stability theory

It is the stability of the planar interface solution of (1.3) to a particular class of perturbations with which we are concerned in the StuartWatson method. After Stuart [13] and Watson [14], we assume that, to third order terms in an amplitude function $A(t)$, the interface satisfies the equation

$$
z=A(t) \cos (\omega x)+A^{2}(t) \zeta_{2}(x)+A^{3}(t) \zeta_{3}(x)+0\left(A^{4}\right)
$$

where $\omega \equiv 2 \pi / \lambda, \lambda$ being the wavelength of the periodic disturbance. The forms of $\zeta_{2}(x)$ and $\zeta_{3}(x)$ can be motivated as follows: To lowest order the interface satisfies the asymptotic relation

$$
z \sim \zeta_{1}(x, t)=A(t) \cos (\omega x)
$$

Upon comparing the forms of

$$
\zeta_{1}^{2}(x, t)=\frac{1}{2} A^{2}(t)[1+\cos (2 \omega x)]
$$

and

$$
\zeta_{1}^{3}(x, t)=\frac{1}{4} A^{3}(t)[3 \cos (\omega x)+\cos (3 \omega x)]
$$

with the second and third terms of (2.1a), respectively, it becomes obvious that we should choose

$$
\zeta_{2}(x)=\zeta_{20}+\zeta_{22} \cos (2 \omega x)
$$

and

$$
\zeta_{3}(x)=\zeta_{31} \cos (\omega x)+\zeta_{33} \cos (3 \omega x)
$$

where the $\zeta_{i j}$ are constants such that each is the coefficient of a term in (2.1) of the form $A^{i}(t) \cos (j \omega x)$ and $\zeta_{11}=1$ while $\zeta_{00}=0$.

We next examine (2.1) for terms proportional to $\cos (\omega x)$ and find them to contain the amplitude functions $A(t)$ and $A^{3}(t)$. Hence we conclude 
that $A(t)$ satisfies the following amplitude equation to third order [5]

$$
\frac{d A}{d t}=\sigma A-a_{1} A^{3}+0\left(A^{5}\right)
$$

where $\sigma$ is the growth rate of linear theory [4] and $a_{1}$ is the Landau constant to be determined. We also assume an analogous expansion for $C(x, z, t)$ consistent with that of $(2.1)$ for $\zeta(x, t)$ where now the coefficient of each term corresponding to a $\zeta_{i j}$ of the latter is considered to be a function of $z$ denoted by $C_{i j}(z)$ with $C_{00}(z)=C_{0}(z)$.

Then after substituting solutions of this type into our governing system of equations (1.1) - (1.4) and expanding the boundary conditions of (1.3) in a Taylor Series about $A=0$, we obtain a set of systems involving an ordinary differential equation in $z$ with boundary conditions evaluated at $z=0$ and as $z \rightarrow \infty$, one for each of the pair of $i$ and $j$ values corresponding to a nonzero term of the form $A^{i}(t) \cos (j \omega x)$ appearing explicitly in (2.1). These are given by

$$
\begin{aligned}
& {\left[D^{2}+D-\left(j^{2} \omega^{2}+i \sigma\right)\right] C_{i j}(z)+a_{1} C_{11}(z) \delta_{i 3} \delta_{1 j}=0, \quad 0<z<\infty ;} \\
& D C_{i j}(0)-(k-1) C_{i j}(0)-(k-1)(k+i \sigma) \zeta_{i j}+a_{1}(k-1) \zeta_{11} \delta_{i 3} \delta_{1 j}=h_{i j}, \\
& C_{i j}(0)+(k-1)\left(1-Q-j^{2} \beta \omega^{2}\right) \zeta_{i j}=b_{i j} \text { with } Q=\frac{G}{M(k-1)} \text { and } \beta=\frac{U}{M(k-1)} \\
& \lim _{z \rightarrow \infty} C_{i j}(z)=0 ;
\end{aligned}
$$

where $\quad D \equiv d / d z, \delta_{i j}=\left\{\begin{array}{ll}1 & i=j \\ 0 & i \neq j\end{array}\right.$, and $h_{11}=b_{11}=0$ while the definitions for the other $h_{i j}$ 's and $b_{i j}$ 's with which we shall be concerned in what follows can be found in the Appendix. Note that $i=j=0$ corresponds to the planar interface solution; hence, the $0(1)$ system is satisfied identically. 
We now catalogue the solutions for the $O(A)$ and $O\left(A^{2}\right)$ systems. In particular the $O(A)$ system which corresponds to $\mathbf{i}=\mathbf{j}=1$ is identical to the linear stability problem of Wollkind and Segel [4] for the special case of equal thermal diffusivities in the liquid and solid phases [11]. Hence from [4], we can conclude that

$$
c_{11}(z)=A_{11} e^{-m_{11} z} \text { and } \zeta_{11}=1
$$

where

$$
A_{11}=-(k-1)\left(1-Q-\beta \omega^{2}\right) \text {, }
$$

and $m_{11}$ is defined implicitly by the relation

$$
m_{i j}^{2}-m_{i j}-\left(j^{2} \omega^{2}+i \sigma\right)=0, \quad \operatorname{Re}\left(m_{i j}\right)>0,
$$

for $i=j=1$, while the eigenvalue $\sigma$ satisfies the following secular equation or dispersion relation

$$
\sigma+k=\left(1-Q-B \omega^{2}\right)\left(m_{11}+k-1\right)
$$

with $m_{11}$ given explicitly by

$$
m_{11}=\frac{1}{2}+\left(\sigma+\omega^{2}+1 / 4\right)^{\frac{1}{2}} \text {. }
$$

The marginal stability curve for this secular equation in the $\omega^{2}-Q$ plane [4],

$$
Q=Q_{c}\left(\omega^{2}\right)=-B \omega^{2}+\frac{\left(1+4 \omega^{2}\right)^{\frac{1}{2}}-1}{2 k-1+\left(1+4 \omega^{2}\right)^{\frac{1}{2}}},
$$

has a maximum at $\omega=\omega_{c}$ where $\omega_{c}$ is defined implicitly by

$$
\beta=\frac{4 k}{\left(1+4 \omega_{c}^{2}\right)^{\frac{1}{2}}\left[2 k-1+\left(1+4 \omega_{c}^{2}\right)^{\frac{1}{2}}\right]^{2}}=f\left(\omega_{c}^{2}\right) .
$$


In what follows, after DiPrima [7], we shall identify the $w$ contained in (2.1) with this $\omega_{c}$, the critical wavenumber of linear stability theory. We further note that consistent with the development already presented in this paper we shall assume $\omega_{c}>k$ or equivalently from (2.9) that

$$
\beta<f\left(k^{2}\right) \text {, }
$$

which guarantees that those $\sigma$ satisfying (2.7) are real valued [15]. Condition (2.10) is valid for the low growth velocities necessary to have neglected the latent heat effect at the interface which is an additional simplification required by both the Sivashinsky model [11] and that of Wollkind and Segel [4]. Hence the assumption of (2.10) is a reasonable one to make within the framework of the model equation under examination.

The $O\left(A^{2}\right)$ systems can be solved in a straightforward manner to yield

$$
c_{2 j}(z)=A_{2 j} e^{-m_{2 j} z} \text { where } A_{2 j}=-(k-1)\left(1-Q-j^{2} \omega^{2} U\right) \zeta_{2 j}+b_{2 j}
$$

and

$$
\zeta_{2 j}=\frac{h_{2 j}+\left(m_{2 j}+k-1\right) b_{2 j}}{(1-k)\left[\left(1-Q-j^{2} \beta \omega^{2}\right)\left(1-k-m_{2 j}\right)+k+2 \sigma\right]}, j=0 \text { and } 2 \text {, }
$$

with $m_{2 j}$ as defined by (2.6c) with $i=2$ and $j=0,2$.

Although there are also two $O\left(A^{3}\right)$ systems, in what follows we shall only be interested in the one corresponding to $i=3$ and $j=1$. In Section 3 we solve this system directly while in Section 4 the indirect adjoint operator method of solvability is employed. Then the results of these two approaches are compared in Section 5 and a final discussion of their relative merits, presented in Section 6 . 
3. The direct method of solution for the $i=3, j=1$ system

In this section we solve the third order system

$$
\begin{gathered}
{\left[D^{2}+D-\left(\omega^{2}+3 \sigma\right)\right] C_{31}(z)=-a_{1} A_{11} e^{-m_{11} z}, \quad z>0 ;} \\
D C_{31}(0)-(k-1) C_{31}(0)-(k-1)(k+3 \sigma) \zeta_{31}+a_{1}(k-1)=h_{31}, \\
C_{31}(0)-A_{11} \zeta_{31}=b_{31} ; \\
\lim _{z \rightarrow \infty} C_{31}(z)=0 ;
\end{gathered}
$$

by what we have termed the direct method. Solving (3.1) for $C_{31}(z ; \sigma)$ by the method of undetermined coefficients and applying the far field boundary condition of (3.3), we obtain

$$
c_{31}(z ; \sigma)= \begin{cases}c_{31}(0 ; \sigma) e^{-m_{31} z}+\frac{a_{1}}{2 \sigma} A_{11}\left(e^{-m_{11} z}-e^{-m_{31} z}\right) & \text { for } \sigma \neq 0 \\ c_{31}(0 ; 0) e^{-m_{01} z}+\frac{a_{1}}{2 m_{01}-1} A_{11} z e^{-m_{01} z} & \text { for } \sigma=0,\end{cases}
$$

where $m_{31}$ and $m_{01}$ satisfy (2.6c) for $i=3, j=1$ and $i=0, j=1$, respectively, and hence are given by

$$
m_{31}=\frac{1}{2}+\left(3 \sigma+\omega^{2}+1 / 4\right)^{\frac{1}{2}} \text { and } m_{01}=\frac{1}{2}+\left(\omega^{2}+1 / 4\right)^{\frac{1}{2}} \text {. }
$$

We first note that since by L'Hospital's Rule

$$
\lim _{\sigma \rightarrow 0} \frac{e^{-m_{11} z}-e^{-m_{31} z}}{2 \sigma}=\frac{z e^{-m_{01} z}}{2 m_{01}-1},
$$

$C_{31}(z ; \sigma)$ is continuous at $\sigma=0$ provided $C_{31}(0 ; \sigma)$ is. 
Further we observe that

$$
D C_{31}(0 ; \sigma)=\left\{\begin{array}{l}
-m_{31} C_{31}(0 ; \sigma)+\frac{a_{1}}{2 \sigma} A_{11}\left(m_{31}-m_{11}\right) \text { for } \sigma \neq 0 \\
-m_{01} C_{31}(0 ; 0)+\frac{a_{1}}{2 m_{01}-1} A_{11} \text { for } \sigma=0
\end{array} .\right.
$$

Then recalling that

$$
m_{31}^{2}-m_{31}-\left(\omega^{2}+3 \sigma\right)=m_{11}^{2}-m_{11}-\left(\omega^{2}+\sigma\right),
$$

we deduce the relationship

$$
\frac{m_{31}-m_{11}}{2 \sigma}=\frac{1}{m_{31}+m_{11}-1}
$$

and hence can conclude that $\mathrm{DC}_{31}(0 ; \sigma)$ is continuous at $\sigma=0$ as well since

$$
\lim _{\sigma \rightarrow 0} m_{31}=\lim _{\sigma \rightarrow 0} m_{11}=m_{01}
$$

Thus with no loss of generality we can represent $D C_{31}(0)$ in $(3.2 a)$ by

$$
D C_{31}(0)=-m_{31} C_{31}(0)+\frac{a_{1}}{m_{31}+m_{11}-1} A_{11}
$$

Then substituting (3.9) into (3.2a), we obtain

$$
a_{1}(k-1)\left[1-\frac{p}{m_{31}+m_{11}-1}\right]-\left(m_{31}+k-1\right) c_{31}(0)-(k-1)(k+3 \sigma) \zeta_{31}=h_{31}
$$

where

$$
P=1-Q-\beta \omega^{2}=\frac{-A_{11}}{k-1} .
$$

Now solving (3.2b) for $c_{31}(0)$,

$$
c_{31}(0)=-(k-1) P \zeta_{31}+b_{31} \text {, }
$$


and substituting (3.12) into (3.10) yields the result

$a_{1}(k-1)\left[1-\frac{p}{m_{31}+m_{11}-1}\right]+(k-1) \zeta_{31}\left[P\left(m_{31}+k-1\right)-k-3 \sigma\right]=h_{31}+b_{31}\left(m_{31}+k-1\right)$.

Since from (2.7a) and (3.11)

$$
\sigma+k=P\left(m_{11}+k-1\right)
$$

then

$$
P\left(m_{31}+k-1\right)-k-\sigma=P\left(m_{31}-m_{11}\right)=2 \sigma \frac{P}{m_{31}+m_{11}-1}
$$

from (3.7). Thus employing (3.15) we obtain the following equation relating $a_{1}$ and $\zeta_{31}$

$$
a_{1}-2 \sigma \zeta_{31}=\frac{h_{31}+b_{31}\left(m_{31}+k-1\right)}{(k-1)\left[1-\frac{p}{m_{31}+m_{11}-1}\right]} \text {, }
$$

from which it is possible to determine the value of the Landau solvability constant $a_{1}$ and then complete the solution by solving for $\zeta_{31}$. Before discussing that procedure in more detail we shall present the indirect adjoint operator approach of determining $a_{1}$. 
4. The adjoint operator method of determining $a_{1}$ We are required to pose and solve the adjoint linear eigenvalue problem as a necessary preliminary step for the application of this method. To do so we must first put the linear eigenvalue problem for $\sigma$ of Section 2--i.e., eqs. (2.3) - (2.5) with $i=j=1--$ in the form [4]

$$
\mathcal{L}\left[\underline{V}_{11}(z)\right]=\sigma M\left[\underline{V}_{11}(z)\right] ;
$$

with boundary conditions such that

$$
B\left[\underline{V}_{11}(0)\right]=0 \text { and } \underline{V}_{11}(z) \rightarrow 0 \text { as } z \rightarrow \infty \text {, }
$$

where $\mathcal{E}$ and $M$ are linear operators on a Hilbert space $H$ consisting of two component vectors

$$
V(z)=[C(z), \zeta]
$$

with the first component having $z \geq 0$ and the second being constant while $B$ is another linear operator which is independent of the eigenvalue $\sigma$ and evaluated at $z=0$. Thus we define

$$
\mathcal{L}[\underline{V}(z)]=\left[\begin{array}{l}
\left(D^{2}+D-\omega^{2}\right) C(z) \\
D C(0)-(k-1) C(0)-k(k-1) \zeta
\end{array}\right], m[\underline{V}(z)]=\left[\begin{array}{l}
C(z) \\
(k-1) \zeta
\end{array}\right],
$$

and

$$
B[\underline{V}(0)]=C(0)+(k-1) P \zeta
$$

while by $\underline{V}(z) \rightarrow 0$ as $z \rightarrow \infty$ and $\underline{V}_{i j}(z)$, we mean $\lim _{z \rightarrow \infty} C(z)=0$ and $\underline{v}_{i j}(z)=\left[C_{i j}(z), \zeta_{i j}\right]$, respectively. (Note: it is not necessary to distinguish between column and row vectors as they will be used interchangeably.) Further we use the inner product, $\langle\underline{U}, \underline{W}>$, of 


$$
\underline{U}=\left[u_{1}(z), u_{2}\right] \text { and } \underline{w}=\left[w_{1}(z), w_{2}\right]
$$

in $H$, defined by

$$
<\underline{U}, \underline{W}>=\int_{0}^{\infty} u_{1}(z) w_{1}(z) d z+u_{2} w_{2} .
$$

Next, we shall pose the adjoint eigenvalue problem

$$
\mathcal{L}^{+}\left[\underline{\mathrm{V}}_{11}^{+}(\mathrm{z})\right]=\mu m^{+}\left[\underline{\mathrm{V}}_{11}^{+}(\mathrm{z})\right]
$$

with boundary conditions on $\underline{v}_{11}^{+}(z)=\left[\mathrm{C}_{11}^{+}(z), \zeta_{11}^{+}\right]$such that

$$
B^{+}\left[\underline{V}_{11}(0)\right]=0 \text { and } \quad \underline{V}_{11}^{+}(z) \rightarrow 0 \text { as } z \rightarrow \infty \text {. }
$$

In addition $\Sigma^{+}, m^{+}$, and $B^{+}$are so defined that for all $\underline{V}$ and $\underline{V}^{+}$ in the respective relevant domains

$$
<\mathcal{L}[\underline{V}(z)], \underline{V}^{+}(z)>=\left\langle\underline{V}(z), \quad \mathcal{L}^{+}\left[\underline{V}^{+}(z)\right]>\right.
$$

and

$$
<m[\underline{V}(z)], \underline{V}^{+}(z)>=\left\langle\underline{V}(z), m^{+}\left[\underline{V}^{+}(z)\right]>\right.
$$

for

$$
\underline{\mathrm{V}}^{+}(z)=\left[\mathrm{C}^{+}(\mathrm{z}), \zeta^{+}\right] \text {in } \mathrm{H}
$$

In order to formulate this problem precisely we need to determine $\Sigma^{+}, \mathrm{m}^{+}$, and $\mathrm{B}^{+}$. Hence we proceed in the following manner: Making use of (4.3) and (4.5), we can write

$$
\begin{aligned}
<\mathcal{L}[\underline{V}(z)], \underline{V}^{+}(z)> & =\int_{0}^{\infty}\left[\left(D^{2}+D-\omega^{2}\right) C(z)\right] C^{+}(z) d z \\
& +[D C(0)-(k-1) C(0)-k(k-1) \zeta] \zeta^{+} .
\end{aligned}
$$

Then upon integrating by parts and using boundary conditions (4.2) as well as the fact that $\lim _{z \rightarrow \infty} C^{+}(z)=0,(4.9)$ becomes 
$<\mathcal{E}[\underline{V}(z)], \underline{V}^{+}(z)>=D C(0)\left[\zeta^{+}-C^{+}(0)\right]-(k-1) \zeta\left(P\left[D C^{+}(0)-C^{+}(0)-(k-1) \zeta^{+}\right]+k \zeta^{+}\right)$

$$
+\int_{0}^{\infty} c(z)\left[\left(D^{2}-D-\omega^{2}\right) C^{+}(z)\right] d z .
$$

Since we wish to define $\mathcal{L}^{+}$and $\mathrm{B}^{+}$such that (4.8a) will be satisfied, we select

$$
\Sigma^{+}\left[\underline{V}^{+}(z)\right]=\left[\begin{array}{l}
\left(D^{2}-D-\omega^{2}\right) C^{+}(z) \\
-(k-1)\left(P\left[D C^{+}(0)-k C^{+}(0)\right]+k \zeta^{+}\right)
\end{array}\right]
$$

and

$$
B^{+}\left[V^{+}(0)\right]=\zeta^{+}-C^{+}(0)=0
$$

Further noting that

$<m[\underline{V}(z)], \underline{V}^{+}(z)>=\int_{0}^{\infty} C(z) C^{+}(z) d z+(k-1) \zeta \zeta^{+}=\left\langle\underline{V}(z), m\left[\underline{V}^{+}(z)\right]>\right.$,

we observe from (4.8b) that

$$
m=m^{+}
$$

hence this operator is formally self-adjoint.

Then employing (4.11) - (4.13) our adjoint eigenvalue problem of (4.6) - (4.7) becomes

$$
\begin{gathered}
{\left[D^{2}-\left(D+\omega^{2}+\mu\right)\right] C_{11}^{+}(z)=0, \quad 0<z<\infty ;} \\
C_{11}^{+}(0)-\zeta_{11}^{+}=0, \\
P\left[-D C_{11}^{+}(0)+k C_{11}^{+}(0)\right]-(k+\mu) \zeta_{11}^{+}=0 ; \\
\lim _{z \rightarrow \infty} C_{11}^{+}(z)=0 .
\end{gathered}
$$

This problem has solution

$$
c_{11}^{+}(z)=\zeta_{11}+e^{-m_{11}^{+} z}
$$

with

$$
\mathrm{m}_{11}^{+}=-\frac{1}{2}+\left(u+\omega^{2}+1 / 4\right)^{1 / 2}
$$


while $\mu$ satisfies

$$
\mu+k=P\left(m_{11}^{+}+k\right)
$$

Since the secular equation (4.18) satisfied by $\mu$ is the same as (3.14) for $\sigma$ because $m_{11}-1=m_{11}^{+}$formally, we can conclude that $\mu=\sigma$; hence we replace $\mu$ in $(4.17)$ by $\sigma$.

Finally we calculate $<m\left[\underline{V}_{11}(z)\right], \underline{v}_{11}{ }^{+}(z)>$ where $\underline{v}_{11}(z)$ is defined by $(2.6)$. That is

$$
<m\left[\underline{\mathrm{V}}_{11}(\mathrm{z})\right], \underline{\mathrm{V}}_{11}^{+}(\mathrm{z})>=\zeta_{11}^{+}(\mathrm{k}-1) \mathrm{F}
$$

where

$$
F=1-\frac{P}{2 m_{11^{-1}}} \text {. }
$$

Traditionally a relation of the form of (4.19) is often used as a normalization condition for the adjoint eigenvector to determine $\zeta_{11}^{+}=\frac{1}{(\mathrm{k}-1) \mathrm{F}}$ but for our purposes it will prove more convenient to take $\zeta_{11}^{+}=1$. Hence our adjoint linear eigenvector is given by

$$
C_{11}^{+}(z)=e^{-\left(m_{11}-1\right) z} \text { and } \zeta_{11}^{+}=1
$$

Having determined this eigenvector, we can now return to the problem at hand. Rewriting the system of (3.1)-(3.3) in the form

$$
\begin{gathered}
\mathcal{L}\left[\underline{V}_{31}(z)\right]-3 \sigma m\left[\underline{V}_{31}(z)\right]+a_{1} m\left[\underline{V}_{11}(z)\right]=\left[\begin{array}{l}
0 \\
h_{31}
\end{array}\right] ; \\
B\left[\underline{V}_{31}(0)\right]=b_{31}, \quad \underline{V}_{31}(z) \rightarrow 0 \text { as } z \rightarrow \infty ;
\end{gathered}
$$

we take the inner product of $(4.21 a)$ with $\underline{v}_{11}^{+}(z)$ of $(4.20)$ and obtain $<\mathcal{E}\left[\underline{V}_{31}(z)\right], \underline{V}_{11}{ }^{+}(z)>-3 \sigma<m\left[\underline{V}_{31}(z)\right], \underline{V}_{11}^{+}(z)>+a_{1}<m\left[\underline{V}_{11}(z)\right], V_{11}{ }^{+}(z)>=h_{31}$. 
Integrating by parts and using (4.21b), one can show that

$$
<\mathcal{E}\left[\underline{V}_{31}(z)\right], \underline{V}_{11}^{+}(z)>=<\underline{V}_{31}(z), \mathcal{L}^{+}\left[\underline{V}_{11}^{+}(z)\right]>-b_{31}\left(m_{11}+k-1\right) .
$$

Since

$$
<\underline{v}_{31}(z), \Sigma^{+}\left[\underline{V}_{11}^{+}(z)\right]>=\left\langle\underline{v}_{31}(z), \sigma m\left[{v_{11}}^{+}(z)\right]>=\sigma<m\left[v_{31}(z)\right], v_{11}^{+}(z)>,\right.
$$

upon substituting the results of (4.19), (4.23), and (4.24) into (4.22), we obtain

$$
a_{1}(k-1) F-2 \sigma<m\left[\underline{V}_{31}(z)\right], \underline{v}_{11}^{+}(z)>=h_{31}+b_{31}\left(m_{11}+k-1\right),
$$

a relation from which $a_{1}$ can readily be determined as we shall demonstrate by the analysis presented in the next section. 
5. Comparison of these two methods

In the last two sections, we derived relations involving the Landau constant $a_{1}$. We now show that both these relations are equivalent and yield the same value for this constant. Specifically we shall deduce (3.16) from (4.25) by using results developed in Section 3. In the spirit of our expression for $D C_{31}(0)$ of (3.9), we first rewrite (3.4) as

$$
c_{31}(z)=c_{31}(0) e^{-m_{31} z}+\frac{a_{1}}{2 \sigma} A_{11}\left(e^{-m_{11} z}-e^{-m_{31} z}\right)
$$

and then, employing (5.1) and (4.20), evaluate

$$
\begin{aligned}
2 \sigma<m\left[\underline{V}_{31}(z)\right], \underline{v}_{11}{ }^{+}(z)>=2 \sigma \int_{0}^{\infty} c_{31}(z) c_{11}^{+}(z) d z+2 \sigma(k-1) \zeta_{31} \zeta_{11}^{+} \\
=\frac{2 \sigma}{m_{31}+m_{11}-1} c_{31}(0)+a_{1} A_{11}\left[\frac{1}{2 m_{11^{-1}}}-\frac{1}{m_{31}+m_{11}-1}\right]+2 \sigma(k-1) \zeta_{31}
\end{aligned}
$$

Using (3.12) and (3.7) we can show that

$$
\frac{2 \sigma}{m_{31}+m_{11}-1} \quad c_{31}(0)=-2 \sigma(k-1) \frac{p}{m_{31}+m_{11}-1} \zeta_{31}+\left(m_{31}-m_{11}\right) b_{31}
$$

Substituting (5.3) into (5.2) and making use of (3.11) and (4.19b), we find that

$$
\begin{gathered}
-2 \sigma<m\left[\underline{v}_{31}(z)\right], \underline{v}_{11}^{+}(z)>=(k-1)\left[a_{1}\left(1-F-\frac{p}{m_{31}+m_{11}-1}\right)-2 \sigma \zeta_{31}\left(1-\frac{p}{m_{31}+m_{11}-1}\right)\right] \\
+b_{31}\left(m_{11}-m_{31}\right) .
\end{gathered}
$$

Finally substitution of (5.4) into (4.25) yields the desired equivalence of the latter to $(3.16)$.

We have deferred until now a discussion of how equation (3.16) is used to determine $a_{1}$ and $\zeta_{31}$. In practice, recalling that we have implicitly taken $\omega=\omega_{c}$ as defined by (2.9), one represents $\sigma$ by the expression [15] 


$$
\sigma=\alpha\left(Q_{c}-Q\right)+O\left(Q-Q_{c}\right)^{2}
$$

where

$$
\alpha^{-1}=\frac{2}{2 k-1+\left(1+4 \omega_{c}^{2}\right)^{\frac{1}{2}}}-\beta>0 \text { and } Q_{c}=Q_{c}\left(\omega_{c}^{2}\right) \text {. }
$$

Then by taking $Q$ sufficiently close to its critical value so that

$$
Q-Q_{C}=O\left(\varepsilon^{2}\right) \text { where }|\varepsilon|<<1 \text {, }
$$

we can guarantee

$$
\sigma=0\left(\varepsilon^{2}\right) \text {. }
$$

Under this condition it can be shown that terms of $O\left(A^{5}\right)$ in the amplitude equation of (2.2) can be neglected [16]. This is the crux of a weakly nonlinear stability theory which, although incorporating the nonlinearities of the problem, basically pivots a perturbation procedure about a critical point of linear stability theory [5]. Also note from (5.5) that

$$
\lim _{Q \rightarrow Q_{C}} \sigma=0 \text { and } \lim _{\sigma \rightarrow 0} Q=Q_{C} \text {. }
$$

In what follows we shall consider all quantities as functions of $\sigma$ and observe from (5.7) that evaluating a quantity at $\sigma=0$ is equivalent to taking $Q=Q_{C}$. In this spirit we symbolically represent (3.16) in the form [3]

$$
a_{1}-2 \sigma \zeta_{31}(\sigma)=r_{31}(\sigma),
$$

where $a_{1}$ is assumed to be constant, $r_{31}(\sigma)$ is the right hand side of (3.16) considered as a function of $\sigma$, and the notation $\zeta_{31}(\sigma)$ is being used to emphasize the explicit dependence of $\zeta_{31}$ on $\sigma$ as well. Now taking the limit of (5.8) as $\sigma \rightarrow 0$ and assuming the requisite continuity at $\sigma=0$, we find

$$
a_{1}=r_{31}(0)
$$


Then upon substitution of this value for the Landau solvability constant into (5.8), we can solve for $\zeta_{31}$ and obtain

$$
\zeta_{31}(\sigma)=\left\{\begin{array}{ll}
\frac{r_{31}(0)-r_{31}(\sigma)}{2 \sigma} & \text { for } \sigma \neq 0 \\
-\frac{1}{2} \frac{d r_{31}(0)}{d \sigma} & \text { for } \sigma=0
\end{array},\right.
$$

assuming the requisite differentiability of $r_{31}(\sigma)$ at $\sigma=0$. Note that $\zeta_{31}(\sigma)$ as determined by $(5.10)$ is continuous at $\sigma=0$ and from (5.6b)

$$
\zeta_{31}(\sigma)=\zeta_{31}(0)+0\left(\varepsilon^{2}\right) \text {. }
$$

Hence equation (3.16) can be used to obtain both $a_{1}$ and $\zeta_{31}$.

Let us now examine how the traditional formulation of $(4.25)$ is used to determine $a_{1}$ and compare the value so obtained with that of (5.9). Representing (4.25) in the form

$$
a_{1}(k-1) F-2 \sigma<m\left[\underline{v}_{31}(z)\right], \underline{v}_{11}^{+}(z)>=S_{31}(\sigma),
$$

we take the limit of (5.12) as $\sigma \rightarrow 0$. Observing from (5.4) that

$$
\lim _{\sigma \rightarrow 0} \sigma<m\left[\underline{V}_{31}(z)\right], \underline{v}_{11}^{+}(z)>=0,
$$

since $\zeta_{31}$ is well behaved at $\sigma=0$, the limits of (3.8) hold, and, in addition,

$$
\lim _{\sigma \rightarrow 0}\left[1-\frac{P}{m_{31}+m_{11}-1}\right]=\lim _{\sigma \rightarrow 0} F=F_{0}=1-\frac{1-Q_{c}-\beta \omega^{2}}{2 m_{01}-1} \text {, }
$$

we then obtain the following value for $a_{1}$ :

$$
a_{1}=\frac{S_{31}(0)}{(k-1) F_{0}}
$$


Using (3.8) and (5.14) in conjunction with the definitions of $r_{31}(\sigma)$ and $S_{31}(\sigma)$ as represented by the right-hand sides of (3.16) and (4.25) respectively, we can readily observe upon comparison of (5.15) to (5.9) that both yield the same value for $a_{1}$, namely,

$$
a_{1}=\frac{h_{31}(0)+b_{31}(0)\left(m_{01}+k-1\right)}{(k-1) F_{0}} \text {, }
$$

which is, of course, not unexpected given the equivalence, as demonstrated above, of the formulations from which they were derived. Hence, equation (4.25) can be used to obtain $a_{1}$ but, unless one converts that equation to (3.16) as we did at the beginning of this section, it is not obvious how one would then determine the solution $\underline{V}_{31}(z)$ from such a formulation. 


\section{Discussion}

Having examined the main principles of both methods, it seems natural to ask which should be employed in a particular instance. If one seeks only the Landau constant itself, the choice of method would most likely depend on the personal preference of the practitioner in question. If, on the other hand, both the Landau constant and a solution to the equations are required, the direct method is superior, since equation (3.16) is essential for these calculations. Although it is possible to obtain this equation using the adjoint operator method, the additional work required to accomplish this is equivalent to simply performing the direct calculation in the first place.

Two points should be brought out at this time. First, the need for both the solution and the Landau constant is not just of academic interest. Problems really do arise in which both are required. Secondly, although the direct method is not mathematically difficult to apply, there are certain subtle features requiring careful attention, or else mistakes can be made. Let us briefly illustrate these ideas with actual examples.

After Segel and Stuart [6], we recently performed a three-dimensional nonlinear stability analysis for a solidification problem in which the interface to lowest order satisfied the equation [3]

$$
z=\zeta(x, y, t) \sim A(t) \cos (\omega x)+B(t) \cos \left(\frac{\omega x}{2}\right) \cos \left(\frac{\sqrt{3} \omega y}{2}\right)
$$

where the amplitude functions $A(t)$ and $B(t)$ were such that

$$
\frac{d A}{d t} \sim \sigma A-a_{0} B^{2}-A\left(a_{1} A^{2}+a_{2} B^{2}\right)
$$

and

$$
\frac{d B}{d t} \sim \sigma B-4 a_{0} A B-B\left(2 a_{2} A^{2}+\frac{a_{1}+2 a_{2}}{4} B^{2}\right) .
$$


Using a direct method of solution identical to that presented in Section 3 we showed that the Landau constants $a_{i} i=0,1,2$ satisfied the following equations of the form of (3.16):

$$
\begin{aligned}
& a_{0}-\sigma \zeta_{0220}(\sigma)=r_{0}(\sigma), \\
& a_{1}=2 \sigma \zeta_{3020}(\sigma)=r_{1}(\sigma),
\end{aligned}
$$

and

$$
a_{2}-2 \sigma \zeta_{1220}(\sigma)=r_{2}(\sigma),
$$

respectively, where $\zeta_{i j p q}$ was the coefficient of a term in the $\zeta$ expansion proportional to $A^{i} B^{j} \cos \left(\frac{p \omega}{2}\right) \cos \frac{(q \sqrt{3} \omega y)}{2}$ and $r_{0}(\sigma)$ was a known function depending on the solutions to the first order systems $(i+j=1)$ while $r_{1}(\sigma)$ and $r_{2}(\sigma)$ were similar functions depending on solutions to the second order systems $(i+j=2)$ as well as on those to first order. Hence, in a completely analogous manner to that presented in Section 5, we found

$$
a_{i}=r_{i}(0) \text { for } i=0,1,2 \text {. }
$$

However since $r_{i}(0) \quad i=1,2$ depended in particular on $\zeta_{0220}(0)$, we had to evaluate this as well and obtained, in the same manner as (5.10) was. deduced, that

$$
\zeta_{0220}(0)=-\frac{d r_{0}}{d \sigma}(0) .
$$

A second illustration can be found in the recent work of Caroli, Caroli and Roulet [17]. It seems appropriate at this point to present a critique of their approach vis-a'-vis our own. Using the approach of [17], one would look for a solution of (3.1) and (3.3) of the form

$$
c_{31}(z)=A_{31} e^{-m 31^{z}}+\frac{a_{1}}{2 \sigma} A_{11} e^{-m} 11^{z} .
$$


Then from (6.6) it follows that

$$
C_{31}(0)=A_{31}+\frac{a_{1}}{2 \sigma} A_{11}
$$

and

$$
D C_{31}(0)=-m_{31} A_{31}-\frac{a_{1}}{2 \sigma} m_{11} A_{11}
$$

Substituting (6.7) into (3.2), one obtains

$$
a_{1}(k-1)-\left(m_{31}+k-1\right) A_{31}-\frac{a_{1}}{2 \sigma} A_{11}\left(m_{11}+k-1\right)-(k-1)(k+3 \sigma) \zeta_{31}=h_{31}
$$

and

$$
A_{31}+\frac{a_{1}}{2 \sigma} A_{11}+(k-1) P_{\zeta_{31}}=b_{31} \text {. }
$$

Eliminating $\frac{a_{1}}{2 \sigma} A_{11}$ between the two equations of (6.8) yields

$$
a_{1}(k-1)+A_{31}\left(m_{11}-m_{31}\right)-2 \sigma(k-1) \zeta_{31}=h_{31}+b_{31}\left(m_{11}+k-1\right),
$$

where use has been made of (3.14) in the above results. Now the approach of [17] entails taking the limit of (6.9) as $\sigma \rightarrow 0$ and obtaining

$$
a_{1}=\frac{s_{31}(0)}{k-1}
$$

under the assumption that

$$
\lim _{\sigma \rightarrow 0} A_{31}\left(m_{11}-m_{31}\right)=0 .
$$

Upon comparing the result of (6.10) with our value for the Landau constant as determined by (5.15), it can be seen that the $a_{1}$ of (6.10) differs from our previous value by a multiplicative factor of $F_{0}$. Caroli, Caroli and Roulet [17] after noting such a discrepancy between their value of $a_{1}$ and that of Wollkind and Segel [4], which had been computed by the adjoint operator method, attributed this to an error on the part of Wollkind and Segel [4] in taking the limit in (5.13) to be zero. Actually their argument is 
fallacious for the following reason. Explicitly denoting the $\sigma$ dependence, eq. (6.7a) implies

$$
A_{31}(\sigma)=C_{31}(0 ; \sigma)-a_{\frac{1}{2 \sigma}} A_{11}(\sigma)
$$

Although it seems perfectly reasonable from a physical standpoint that $C_{31}(0 ; \sigma)$ and $\zeta_{31}(\sigma)$ would be well behaved in the limit as $\sigma \rightarrow 0$, the same is not true for $A_{31}(\sigma)$ which becomes unbounded in that limit. Indeed employing (6.12), (3.7), and (3.11)

$$
A_{31}(\sigma)\left(m_{11}-m_{31}\right)=c_{31}(0 ; \sigma)\left(m_{11}-m_{31}\right)-\frac{(k-1) P}{m_{31}+m_{11}-1} a_{1}
$$

Hence taking the limit of (6.13) as $\sigma \rightarrow 0$, we obtain

$$
\lim _{\sigma \rightarrow 0} A_{31}(\sigma)\left(m_{11}-m_{31}\right)=\left(F_{0}-1\right)(k-1) a_{1} .
$$

Now by taking the limit of (6.9) as $\sigma \rightarrow 0$ and using this condition of (6.14) instead of $(6.11)$, a value of $a_{1}$ results which is equal to that of (5.15). Thus the approach of Caroli, Caroli and Roulet [17] when interpreted correctly is a hybridization of our direct method of solution and the special case of that method presented below.

It is instructive to note that the following version of the direct method with $\sigma$ taken to be equal to zero from the outset is probably the simplest way to compute Landau-type constants. Consider, for example, the third order system, (3.1) - (3.3), in the limit as $\sigma \rightarrow 0$. Then, from (3.6), taking

$$
D C_{31}(0)=-m_{01} C_{31}(0)+\frac{a_{1}}{2 m_{01}-1} A_{11}(0)
$$


where

$$
P_{0}=1-Q_{c}-\beta \omega^{2}=-\frac{A_{11}(0)}{k-1},
$$

and substituting into (3.2a), we obtain

$$
a_{1}(k-1) F_{0}-\left(m_{01}+k-1\right) c_{31}(0)-k(k-1) \zeta_{31}(0)=h_{31}(0)
$$

Now using (3.12) to eliminate $C_{31}(0)$ from $(6.17)$, the latter becomes

$$
a_{1}(k-1) F_{0}+(k-1) \zeta_{31}(0)\left[P_{0}\left(m_{01}+k-1\right)-k\right]=h_{31}(0)+b_{31}(0)\left(m_{01}+k-1\right)=s_{31}(0) \text {. }
$$

Then noting, either from (3.14) in the limit as $\sigma \rightarrow 0$ or from the definition of $Q_{C}$ given by (2.8), that

$$
k=P_{0}\left(m_{01}+k-1\right),
$$

the term proportional to $\zeta_{31}(0)$ in (6.18) disappears and the result of (5.15) follows immediately.

In summary, we have presented two different methods for determining the Landau constant in the amplitude equation for a weakly nonlinear stability analysis of a model system for alloy solidification. Our analysis indicates that, for such a Stefan-type moving boundary problem, what we have termed the direct method is preferable to the more traditional adjoint operator method of computing the Landau constant.

We close by offering one final additional advantage of employing the direct method for calculating the Landau constant as opposed to using the adjoint operator method instead and that is the former requires less mathematical sophistication then does the latter when applied to Stefan-type moving boundary problems. Historically Stefan problems involving parabolic diffusion 
equations which must be satisfied in a region or regions whose boundaries are to be determined have been mainly important in the fields of fluid mechanics [18] and metallurgy [1 ]. Now we see examples in the textile, glass, and chemical industries as well as in the fields of geophysics, meteorology, biology, and astrophysics [2]. With so many researchers from varied backgrounds potentially interested in solving the type of problem under examination in this paper, there is some merit in introducing the simplest reasonable method of solution in terms of the mathematical sophistication required before one feels both at relative ease in applying, and confident using the technique in question. Indeed, Caroli, Caroli and Roulet's [17] rationale for employing their version of the direct method was that by doing so they could bypass the details of the adjoint operator method used by Wollkind and Segel [4] It is in this spirit that the direct method of solution presented in this paper should be employed and possibly such employment may make nonlinear stability analyses more accessible to a wider class of researchers than has been the case heretofore with the adjoint operator method. 


\section{Acknowledgment}

David Wollkind would like to thank the Institute for Mathematics and its Applications at the University of Minnesota and its director Professor Hans Weinberger for providing both support and an atmosphere conducive to producing research during the preparation of this manuscript. 
Appendix

The nonhomogeneous terms appearing in (2.4) are given by

$$
\begin{aligned}
& h_{20}=\frac{1}{2}\left[-D^{2} C_{11}(0)+(k-1) D C_{11}(0)+\left(\sigma[k-1]+\omega^{2}\right) C_{11}(0)\right] \zeta_{11}+\frac{k-1}{2}\left[\sigma(k-1)-\frac{k}{2}\right] \zeta_{11}^{2}, \\
& h_{22}=\frac{1}{2}\left[-D^{2} C_{11}(0)+(k-1) D C_{11}(0)+\left(\sigma[k-1]-\omega^{2}\right) C_{11}(0)\right] \zeta_{11}+\frac{k-1}{2}\left[\sigma(k-1)-\frac{k}{2} \zeta_{11}^{2},\right. \\
& b_{20}=b_{22}=\frac{1}{2} \zeta_{11}\left[\frac{k-1}{2} \zeta_{11}-D C_{11}(0)\right], \\
& h_{31}=k(k-1) \zeta_{11}\left[\frac{1}{8} \zeta_{11}^{2}-\left(\zeta_{20}+\frac{1}{2} \zeta_{22}\right)\right]-\left[D^{2} C_{11}(0)-(k-1) D C_{11}(0)\right]\left(\zeta_{20}+\frac{1}{2} \zeta_{22}\right) \\
& \quad-\frac{3}{8} \zeta_{11}^{2}\left[D^{3} C_{11}(0)-(k-1) D^{2} C_{11}(0)\right]-\zeta_{11}\left(\left[D^{2} C_{20}(0)-(k-1) D C_{20}(0)\right]\right. \\
& \left.+\frac{1}{2}\left[D^{2} C_{22}(0)-(k-1) D C_{22}(0)\right]\right)+\omega^{2}\left[C_{11}(0) \zeta_{22}+C_{22}(0) \zeta_{11}+\frac{1}{4} D C_{11}(0) \zeta_{11}^{2}\right] \\
& +\sigma(k-1)\left(\zeta_{11}\left[C_{20}(0)+\frac{1}{2} C_{22}(0)+3(k-1)\left(\zeta_{20}+\frac{1}{2} \zeta_{22}\right)+\frac{3}{4} \zeta_{11}\left\{D C_{11}(0)-\frac{k-1}{2} \zeta_{11}\right\}\right]\right. \\
& \left.+C_{11}(0)\left[2 \zeta_{20}+\zeta_{22}\right]\right),
\end{aligned}
$$

and

$$
\begin{aligned}
b_{31} & =(k-1) \zeta_{11}\left(\zeta_{20}+\frac{1}{2} \zeta_{22}\right)-\frac{k-1}{8} \zeta_{11}^{3}-D C_{11}(0)\left(\zeta_{20}+\frac{1}{2} \zeta_{22}\right) \\
& -\frac{3}{8} D^{2} C_{11}(0) \zeta_{11}^{2}-\zeta_{11}\left[D C_{20}(0)+\frac{1}{2} D C_{22}(0)\right]-\frac{3}{8}(k-1) B \omega^{4} \zeta_{11}^{3} .
\end{aligned}
$$


References

[1] M. C. Flemings, Solidification Processing (McGraw-Hi11, New York, 1974).

[2] J. R. Ockendon and W. R. Hodgkins, Moving Boundary Problems in Heat Flow and Diffusion (Clarendon Press, 0xford, 1975).

[3] R. Sriranganathan, D. J. Wollkind and D. B. Oulton, J. Crystal Growth (in press).

[4] D. J. Wollkind and L. A. Segel, Phil. Trans. Roy. Soc. London 268(1970) 351.

[5] P. G. Drazin and W. H. Reid, Hydrodynamic Stability (Cambridge University Press, Cambridge, 1981).

[6] L. A. Segel and J. T. Stuart, J. Fluid Mech. 13(1962) 289.

[7] R. C. DiPrima, Vector eigenfunction expansions for growth of Taylor vortices in the flow between rotating cylinders, Nonlinear Partial Differential Equations (Academic Press, New York, 1967).

[8] W. Eckhaus, Studies in Non-Linear Stability Theory (Springer, New York, 1965).

[9] D. D. Joseph, Stability of Fluid Motions II (Springer, New York, 1976).

[10] L. A. Segel, Nonlinear problems in hydrodynamic stability, Nonequilibrium Thermodynamics, Variational Techniques, and Stability (University of Chicago Press, Chicago, 1966).

[11] G. I. Sivashinsky, Physica D (in press).

[12] J. S. Langer, Rev. mod. Phys. 52 (1980) 1.

[13] J. T. Stuart, J. Fluid Mech. 9 (1960) 353. 
[14] J. Watson, J. Fluid Mech. 9 (1960) 371.

[15] D. J. Wollkind, A. deterministic continuum mechanical approach to morphological stability of the solid-liquid interface, Preparation and Properties of Solid State Materials, Vol. 4 (Marcel Dekker, New York, 1979) .

[16] B. J. Matkowsky, Bu11. Amer. Math. Soc. 76 (1970) 620.

[17] B. Caroli, C. Caroli and B. Roulet, J. Physique (in press).

[18] G. K. Batchelor, An Introduction to Fluid Dynamics (Cambridge University Press, Cambridge, 1967). 


\section{Recent IMA Preprints}

(cont inued)

15

16
K.A. Pericak-Spector

George R. Sell
On Stability and Uniqueness of Fluid Flow Through a Rigid Porous Medium

Smooth Linearization Near a Fixed Point 